\title{
Reliability of a Caton-Deschamps-derived patella height index for knee arthroplasty
}

\author{
Christian Konrads $^{1} \cdot$ Lucia C. Grosse ${ }^{1} \cdot$ Sufian S. Ahmad ${ }^{1,2} \cdot$ Fabian Springer $^{3} \cdot$ Anna J. Schreiner $^{1}$. \\ Florian Schmidutz ${ }^{1,4} \cdot$ Felix Erne $^{1}$
}

Received: 23 August 2020 / Accepted: 29 December 2020 / Published online: 20 January 2021

(C) The Author(s) 2021

\begin{abstract}
Purpose The original Caton-Deschamps index (oCDI) detects functional patella height. It cannot be used in knees with an implanted endoprosthesis. The "modified Caton-Deschamps index" (mCDI) for knee arthroplasty can miss pseudo-patellainfera (PPI), which is common after TKA. A derivate of the oCDI could be a simple analogue to the index published in 1982 using a modified tibial reference point at the anterior proximal point of the inlay, which can indirectly be located on the lateral knee radiograph. It was the aim of this study to determine the intra- and inter-rater agreement of a derived Caton-Deschamps index (dCDI) for knee arthroplasty. We hypothesized that the derived Caton-Deschamps index (dCDI) is a reliable radiological measure for patella height in knee arthroplasty.

Methods Several patella height indices were measured by three independent raters in two passes. The second pass was performed after 6 weeks in random order. Intra- and inter-observer agreements were determined and analyzed using the intraclass correlation coefficient (ICC). For radiographic evaluation, digital lateral radiographs of 150 knees before and after primary TKA were used. Results We found high interrater reliability for all analyzed indices. We found the highest agreements for the ISI preop (ICC = 0.914 ) and postop (ICC $=0.920$ ), respectively. We also found very good intra-rater reliability for the CDI (ICCpreop $=0.954)$, $\mathrm{dCDI}($ ICCpostop $=0.945)$, ISI $($ ICCpreop $=0.960$; ICCpostop=0.940) and BPI $($ ICCpreop $=0.969$; ICCpostop $=0.955)$. Fourteen cases $(9.3 \%)$ with insignificant PPI were found.

Conclusion The derived Caton-Deschamps index (dCDI) can easily be used in knee arthroplasty and demonstrated high intraand interrater agreement, which was similar to other commonly used and established patella height indices.
\end{abstract}

Keywords TKA $\cdot$ Patella height $\cdot$ Patella infera $\cdot$ Patella baja $\cdot$ Anterior knee pain $\cdot$ Insall-Salvati

\section{Introduction}

Measuring patella height has been a hot topic since decades [1-4]. Multiple radiological indices exist for native knees and for arthroplasty [5-7]. True patella height is determined by the

Christian Konrads

christian.konrads@gmail.com

1 Department for Trauma and Reconstructive Surgery, BG Klinik, University of Tübingen, Tübingen, Germany

2 Center for Musculoskeletal Surgery, Charité - University Medical Center Berlin, Berlin, Germany

3 Department of Radiology, BG Klinik, University of Tübingen, Tübingen, Germany

4 Department of Orthopaedic Surgery, Physical Medicine and Rehabilitation, University of Munich (LMU), Munich, Germany length of the patellar ligament. Pseudo patella height alteration is a relative alteration of the patella height in relation to the femoro-tibial joint line without change in the length of the patellar ligament [8]. Proximalisation of the joint line can be accidentally created during total knee arthroplasty (TKA) leading to pseudo-patella-infera (PPI) [9-11]. Patella infera can be a combination of TPI and PPI. Both components should be part of a complete patella height assessment, which demands a combination of patella height indices [12-14]. If only one index is to be used, it should be made sure that this index can completely detect the sought-after patella height pathology.

The Insall-Salvati index (ISI) detects true patella height only [15]. The original Caton-Deschamps index (CDI) published in 1982 is commonly accepted as a measure of functional patella height $[3,16]$. As the tibial landmark used for this index is being resected during knee arthroplasty and as the tibial inlay is not visible on X-ray due to its radiolucency, the 
index in its original form cannot be used in knees with an implanted endoprosthesis. The "modified Caton-Deschamps index" (mCDI) for knee arthroplasty published in 2016 can miss the detection of PPI. This is a problem as PPI is common after TKA [17].

A derivate of the original CDI could be a simple analogue to the index published in 1982 using a modified tibial reference point at the anterior proximal point of the inlay, which can indirectly be found on the lateral knee radiograph (Fig. 1). It was the aim of this study to determine the intra- and interrater agreement of a derived Caton-Deschamps index (dCDI) for knee arthroplasty.

We hypothesize that the derived Caton-Deschamps index $(\mathrm{dCDI})$ is a reliable radiological measure for patella height in knee arthroplasty.

\section{Patients and methods}

From our database, we retrospectively identified the patients who received a primary bicondylar standard TKA in 2015. Randomly, 150 patients were chosen.

All operations were performed by experienced surgeons and strictly according to internal standard operation procedures. Peri-operatively, an intravenous medication with $2 \mathrm{~g}$ Cefazolin was applied as a single shot. All operations were controlled by computer navigation using the Orthopilot TKA software. After median skin incision, an anteromedial parapatellar arthrotomy was performed. The Hoffa fat pad was partially resected. A patellar denervation with resection of osteophytes was done as part of the standard procedure. The rotation of the femoral component was oriented in relation to the transepicondylar axis. Prior to the cemented implantation of tibial and femoral components, the pneumatic tourniquet was applied, and bone surface was prepared by Jet Lavage. In addition to the software protocol, a manual control of range of motion and patella tracking were performed. A closed suction drainage was applied. In preparation of wound closure, local anaesthetics and tranexamic acid were injected. After wound closure, a sterile dressing was applied.

All patients were mobilized with allowed full weightbearing at the first day after surgery. The suction drainage was removed at the second day after surgery. Patients received physiotherapy daily. Discharge was 7.0 days after surgery. A three week rehab was organized. All patients had a postoperative follow-up appointment about three, six, 12 and 24 months after surgery for clinical and radiographic control.

We chose the following radiological indices for patella height measurements on the lateral radiographs:

- Insall-Salvati index (ISI) $[15,18]$

- Modified Insall-Salvati index (mISI) [19]

- Caton-Deschamps index [16, 20] (CDI) pre-operatively and a derived Caton-Deschamps index [6] (dCDI) postoperatively (Fig. 2)

- Blackburne-Peel index (BPI) [21]

Values for classification into groups of patella infera, norma, or alta are shown in Table 1.

The radiographic indices were measured on lateral radiographs of the knee independently by three raters (one inexperienced but trained rater, one experienced orthopaedic surgeon and one experienced musculoskeletal radiologist). For determination of not only the interrater reliability, but also the intra-
Fig. 1 Caton-Deschamps index for native knees (a) and derived Caton-Deschamps index for arthroplasty $(\mathbf{b})$. CDI $=$ CatonDeschamps index; $\mathrm{dCDI}=\mathrm{de}-$ rived Caton-Deschamps index
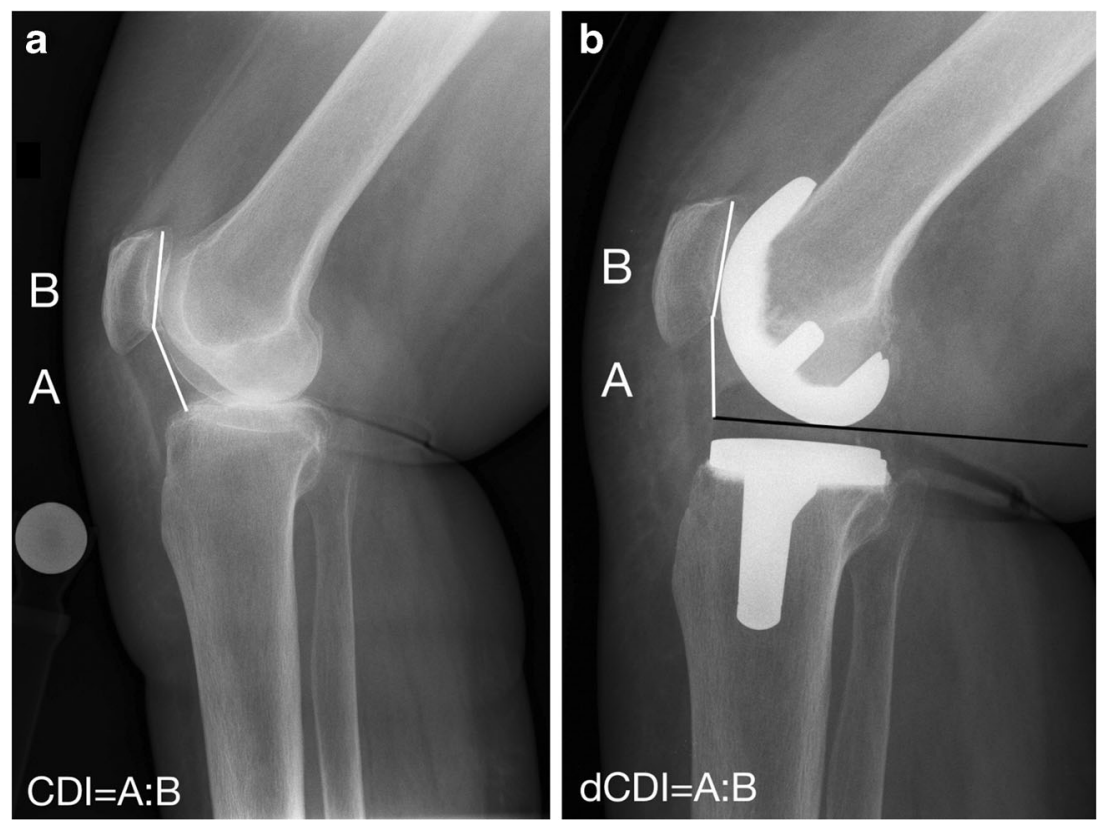


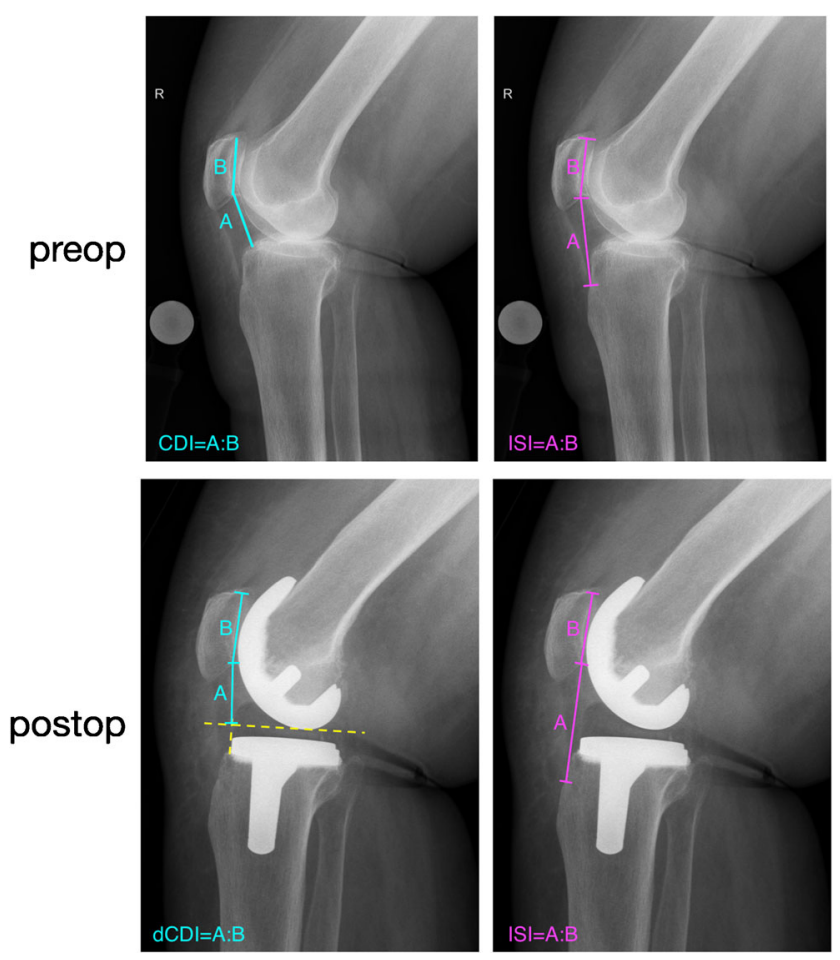

Fig. 2 Essential indices for patellar-height analysis determining true patella height and pseudo patella height alterations. ISI = Insall-Salvati index, $\mathrm{CDI}=$ Caton-Deschamps index, $\mathrm{dCDI}=$ derived CatonDeschamps index (modification of the CDI for arthroplasty)

rater reliability, all measures were repeated after six weeks. This was done in a randomized order to eliminate any bias from the first reading.

Intra- and inter-observer agreements were determined and analyzed using the intraclass correlation coefficient (ICC).

Ethical approval was received for the conduction of this study by our university's review board.

For measuring patella height in knee arthroplasty using the $d C D I$, a tibial reference point at the superior anterior part of the inlay was used. Like in the oCDI, this point lies on the femoro-tibial joint line. It is identified on a lateral knee radiograph by drawing a tangential line to the femoral component. This line is parallel to the tibial component (Fig. 1).

For comparison of the dCDI with the mCDI, we additionally measured the $\mathrm{mCDI}$ in ten cases with post-operative PPI.

Table 1 Patella height indices

\begin{tabular}{llll}
\hline Index & Patella infera & Patella norma & Patella alta \\
\hline Insall-Salvati & $<0.8$ & $0.8-1.2$ & $>1.2$ \\
Modified Insall-Salvati & $<1.2$ & $1.2-2.0$ & $>2.0$ \\
Caton-Deschamps & $<0.6$ & $0.6-1.2$ & $>1.2$ \\
Blackburne-Peel & $<0.6$ & $0.6-1.0$ & $>1.0$ \\
\hline
\end{tabular}

\section{Results}

Out of 150 patients receiving primary TKA, 82 were females and 68 were males. Seventy-eight were right knees and 72 were left knees. The mean age of patients receiving TKA was 73 (52-88).

Testing the reliability of the derived Caton-Deschamps index (dCDI) as a radiological measure for patella height in knee arthroplasty revealed the following results:

We demonstrated high inter-rater reliability for all analyzed indices (Table 2). We found the highest agreements for the ISI preop and postop, respectively. We also found very good intra-rater reliability for the CDI, dCDI, ISI and BPI (Table 3).

Comparing pre-operative and post-operative radiographs, we found TPI, which did not exist pre-operatively, in $2.2 \%$ of the cases post-operatively. PPI was found in $9.8 \%$ after TKA.

In cases with post-operative PPI, the preop oCDI was 0.76 \pm 0.13 and the postop dCDI was $0.54 \pm 0.12$ on average, whereas the mCDI was $1.14 \pm 0.24$ pre-operatively and 1.03 \pm 0.28 post-operatively.

\section{Discussion}

Patella infera after TKA is a known complication [13, 22]. Direct shortening of the patellar ligament is called TPI and probably occurs due to scarring during the first two years after surgery [22]. It can detoriate clinical outcome leading to anterior knee pain and reduced range of motion. PPI is a common finding after TKA, and within a certain unknown range, it is most likely not correlated with reduced clinical outcome $[8$, 9].

Given the fact that patella height assessment is under debate since decades and multiple indices for measuring patella height exist, a reliable concept for determining patella height not only in native knees but also in knee arthroplasty is needed [23]. Therefore, we asked the question, if the original CatonDeschamps index (CDI) could also reliably be used in knee arthroplasty utilizing a modified tibial reference point at the proximal anterior part of the inlay (Fig. 1). This would make comparison of pre-operative and post-operative patella height

Table 2 Inter-rater reliability (ICC) for several patella height indices

\begin{tabular}{lllll}
\hline Rater & $\begin{array}{l}\text { CDI before } \\
\text { TKA }\end{array}$ & $\begin{array}{l}\text { dCDI after } \\
\text { TKA }\end{array}$ & $\begin{array}{l}\text { ISI before } \\
\text { TKA }\end{array}$ & $\begin{array}{l}\text { ISI after } \\
\text { TKA }\end{array}$ \\
\hline Rater 1-rater 2 & 0.952 & 0.912 & 0.952 & 0.936 \\
Rater 1-rater 3 & 0.863 & 0.818 & 0.893 & 0.924 \\
Rater 2-rater 3 & 0.891 & 0.826 & 0.898 & 0.901 \\
\hline
\end{tabular}

ICC intraclass correlation coefficient, $C D I$ Caton-Deschamps index, $d C D I$ derived Caton-Deschamps index, ISI Insall-Salvati index, TKA total knee arthroplasty 
Table 3 Intra-rater reliability (ICC) for several patella height indices

\begin{tabular}{lllllll}
\hline Rater & $\begin{array}{l}\text { CDI before } \\
\text { TKA }\end{array}$ & $\begin{array}{l}\text { dCDI after } \\
\text { TKA }\end{array}$ & $\begin{array}{l}\text { ISI before } \\
\text { TKA }\end{array}$ & $\begin{array}{l}\text { ISI after } \\
\text { TKA }\end{array}$ & $\begin{array}{l}\text { BPI before } \\
\text { TKA }\end{array}$ & $\begin{array}{l}\text { BPI after } \\
\text { TKA }\end{array}$ \\
\hline Rater 1 & 0.926 & 0.918 & 0.956 & 0.947 & 0.969 & 0.955 \\
Rater 2 & 0.982 & 0.972 & 0.965 & 0.993 & & \\
\hline
\end{tabular}

$I C C$ intraclass correlation coefficient, $B P I$ Blackburne-Peel index, $C D I$ Caton-Deschamps index, $d C D I$ derived Caton-Deschamps index, ISI Insall-Salvati index, TKA total knee arthroplasty feasible using the original CDI pre-operatively and a derived CDI after TKA, for recording the full amount of potential patella infera, which could consist of TPI and PPI. The index that we call dCDI has been used before, but to our best knowledge, it has never been completely analyzed regarding its reliability.

We demonstrated high intra- and inter-observer agreement for the dCDI. The reliability of the dCDI was very similar to the most accepted patella height indices: ISI, BPI and CDI (Tables 2 and 3) [23]. So, patella height measured using the CDI preoperatively can now be compared with the postoperative situation after TKA using the dCDI. Normal values for the dCDI should be the same as for the original CDI, but validation remains pending.

An advantage of the dCDI over other indices is that it can fully detect patella height in knee arthroplasty and it can be used very similar to the oCDI, which is utilized in native knees. Therefore, the dCDI fits perfectly in our concept of patella height assessment, which is depicted in Fig. 2. It is easy to use and differentiation between PPI and TPI is possible.

As the $\mathrm{dCDI}$ is referenced at the femoro-tibial joint-line, it detects any patella-height alteration, which could happen also in revision TKA with change of any or all implants. In contrast, the mCDI published in 2016 would remain unchanged in cases of revision with inlay exchange to a different height for example.

This study had a number of limitations. First, it was a retrospective design. Second, the number of knees were limited. Third, the number of observers were limited. Despite these limitations, given that the aim of this study was to prove the concept, the authors agreed on the sufficiency of the design chosen in this study.

\section{Conclusions}

The derived Caton-Deschamps index (dCDI) can easily be used in knee arthroplasty and demonstrated high intra- and inter-rater agreement, which was similar to other commonly used and established patella height indices. For functional patella height assessment, the CDI can be used preoperatively and the dCDI post-operatively. For full analysis of PPI and TPI, the ISI can be used additionally to determine the amount of TPI, while the amount of PPI can then be calculated. The normal range of the $\mathrm{dCDI}$ is most likely the same as for the CDI, but this should be verified and validated in a future study with clinical correlation.

Funding Open Access funding enabled and organized by Projekt DEAL.

Data availability The datasets generated and analyzed during the current study are available from the authors on reasonable request.

\section{Compliance with ethical standards}

Conflict of interest The authors declare that they have no conflict of interest.

Ethics approval Yes.

Consent to participate Yes.

Consent for publication Yes.

Open Access This article is licensed under a Creative Commons Attribution 4.0 International License, which permits use, sharing, adaptation, distribution and reproduction in any medium or format, as long as you give appropriate credit to the original author(s) and the source, provide a link to the Creative Commons licence, and indicate if changes were made. The images or other third party material in this article are included in the article's Creative Commons licence, unless indicated otherwise in a credit line to the material. If material is not included in the article's Creative Commons licence and your intended use is not permitted by statutory regulation or exceeds the permitted use, you will need to obtain permission directly from the copyright holder. To view a copy of this licence, visit http://creativecommons.org/licenses/by/4.0/.

\section{References}

1. Gracitelli GC, Pierami R, Tonelli TA et al (2012) Assessment of patellar height measurement methods from digital radiography. Rev Bras Ortop 47:210-213

2. Narkbunnam R, Chareancholvanich K (2015) Effect of patient position on measurement of patellar height ratio. Arch Orthop Trauma Surg 135:1151-1156

3. van Duijvenbode D, Stavenuiter M, Burger B, van Dijke C, Spermon J, Hoozemans M (2016) The reliability of four widely used patellar height ratios. Int Orthop 40:493-497

4. Yiannakopoulos CK, Mataragas E, Antonogiannakis E (2008) The effect of quadriceps contraction during weight-bearing on four patellar height indices. J Bone Joint Surg (Br) 90:870-873 
5. Philips CL, Silver DAT, Schranz PJ, Mandalia V (2010) The measurement of patellar height: a review of the methods of imaging. Bone Joint J 92:1045-1053

6. Xu B, Xu W-X, Lu D, Sheng H-F, Xu X-W, Ding W-G (2017) Application of different patella height indices in patients undergoing total knee arthroplasty. J Orthop Surg Res 12:191

7. Yuan F, Sun Z, Wang H, Chen Y, Yu J (2019) Clinical and radiologic outcomes of two patellar resection techniques during total knee arthroplasty: a prospective randomized controlled study. Int Orthop 3:2293-2301

8. Bugelli G, Ascione F, Cazzella N et al (2018) Pseudo-patella baja: a minor yet frequent complication of total knee arthroplasty. Knee Surg Sports Traumatol Arthrosc 26:1831-1837

9. Aguirre-Pastor A, Ortolá DJ, Lizaur-Utrilla A, Rosa MA, LopezPrats FA (2020) Is pseudo-patella baja really a serious complication of total knee arthroplasty? J Arthroplast 32:557-562

10. Prudhon JL, Caton JH, Aslanian T, Verdier R (2018) How is patella height modified after total knee arthroplasty? Int Orthop 42:311316

11. Schiapparelli F-F, Ahmadi P, Amsler F, Hirschmann MT (2020) No reduced patellar loading with latest-generation cruciateretaining total knee arthroplasty: a comparative study of Attune and Press-Fit Condylar. Int Orthop 44:online ahead of print

12. Chonko DJ, Lombardi AVJ, Berend KR (2004) Patella baja and total knee arthroplasty (TKA): etiology, diagnosis, and management. Surg Technol Int 12:231-238

13. Lum ZC, Saiz AM, Pereira GC, Meehan JP (2020) Patella baja in total knee arthroplasty. J Am Acad Orthop Surg 28:316-323

14. Sundararajan SR, Raj M, Ramakanth R, Muhil K, Rajasedaran S (2020) Prediction of recurrence based on the patellofemoral morphological profile and demographic factors in first-time and recurrent dislocators. Int Orthop 2020 44: online ahead of print

15. Insall J, Salvati E (1972) Patella position in the normal knee joint. Radiology 101:101-104

16. Caton J, Deschamps G, Chambat P, Lerat JL, Dejour H (1982) Patella infera. Apropos of 128 cases. Rev Chir Orthop Reparatrice Appar Mot 68:317-325

17. Caton JH, Prudhon JL, Aslanian T, Verdier R (2016) Patellar height assessment in total knee arthroplasty: a new method. Int Orthop 40: $2527-2531$

18. Verhulst FV, van Sambeeck JDP, Olthuis GS, van der Ree J, Koëter S (2020) Patellar height measurements: Insall-Salvati ratio is most reliable method. Knee Surg Sports Traumatol Arthrosc 28:869-875

19. Grelsamer RP, Meadows S (1992) The modified Insall-Salvati ratio for assessment of patellar height. Clin Orthop Relat Res 282:170 176

20. Dejour DH (2013) The patellofemoral joint and its historical roots: the Lyon School of Knee Surgery. Knee Surg Sports Traumatol Arthrosc 21:1482-1494

21. Blackburne JS, Peel TE (1977) A new method of measuring patellar height. J Bone Joint Surg (Br) 59:241-242

22. Davies GS, van Duren B, Shorthose M et al (2016) Changes in patella tendon length over 5 years after different types of knee arthroplasty. Knee Surg Sports Traumatol Arthrosc 24:3029-3035

23. Cabral F, Sousa-Pinto B, Pinto R, Torres J (2017) Patellar height after total knee arthroplasty: comparison of 3 methods. J Arthroplast 32:552-557

Publisher's note Springer Nature remains neutral with regard to jurisdictional claims in published maps and institutional affiliations. 